\title{
Ultrasound diagnosis and neurodevelopmental outcome of localised and extensive cystic periventricular leucomalacia
}

\author{
V Pierrat, C Duquennoy, I C van Haastert, M Ernst, N Guilley, L S de Vries
}

\begin{abstract}
Aims-To compare the ultrasound (US) evolution and neurodevelopmental outcome of infants with localised (grade II) and extensive (grade III) cystic periventricular leucomalacia (c-PVL).

Methods-Over a nine year period, c-PVL was diagnosed in $96 / 3451(2.8 \%)$ infants in two hospital cohorts. Eighteen were excluded from the study. Thirty nine infants with grade II PVL were compared with 39 infants with grade III PVL.
\end{abstract}

Results-The two populations were comparable for gestational age and birth weight. In infants with grade II PVL, cysts were noted to develop more often after the first month of life $(53 \%)$ in contrast with grade III PVL (22\%) (odds ratio (OR) 3.81 (95\% confidence interval (CI) 1.19 to 12.63)). Cysts were also more often unilateral in grade II (54\%) than in grade III PVL (0\%) (OR indefinite; RR 3.17 (95\% CI 2.16 to 4.64$)$ ). At 40 weeks postmenstrual age (PMA), cysts were no longer seen on US in 13/38 infants with grade II PVL, with ventriculomegaly being the only visible sequel in nine cases. In grade III PVL, cysts were still present in 25 of the 27 surviving infants. Nine infants with grade II PVL were free of motor sequelae at follow up compared with one infant with grade III PVL (OR 8.07 (95\% CI 0.92 to 181.66)). Twenty two out of 29 children with grade II PVL who developed cerebral palsy achieved independent walking compared with 3/26 with grade III PVL (OR 75 (95\% CI 11.4 to 662)).

Conclusions-In the cohort studied, $50 \%$ of the infants with c-PVL had a more localised form (grade II). In grade II PVL, the cysts developed beyond the first month of life in more than half of the cases and were often no longer visible, on US, at 40 weeks PMA. In order not to miss this diagnosis, sequential US should also be performed beyond the first month of life. Mild ventriculomegaly noted at term can sometimes be due to grade II c-PVL. Cerebral palsy was slightly less common and tended to be less severe in infants with grade II PVL than in those with grade III PVL. (Arch Dis Child Fetal Neonatal Ed 2001;84:F151-F156)
Keywords: periventricular leucomalacia; ultrasound; neurodevelopmental outcome; ventriculomegaly; cerebral palsy

The diagnosis of periventricular leucomalacia (PVL) was initially only made when areas of increased echogenicity on cranial ultrasound (US) evolved into cystic lesions. ${ }^{12}$ Subsequently, prolonged "flares" were considered to be a milder degree of PVL. More recently, attention has been paid to ventriculomegaly, and it was suggested that ventriculomegaly on its own could be a marker of white matter disease. ${ }^{34}$ Cystic PVL (c-PVL), referred to in the literature, is usually extensive c-PVL (grade III according to de Vries et $a \bar{l}$ ). The presence of smaller and more localised cysts (grade II according to de Vries $e t a \bar{l}$ ) is less commonly reported. Fawer and Calame ${ }^{6}$ were the first to report the diagnosis of localised c-PVL and the subsequent neurological development in a small group of preterm infants. Evolution of the US lesions in PVL grade II has, however, not yet been reported in detail.

Initial follow up studies ${ }^{7-10}$ reported the outcome of children with c-PVL, irrespective of the site, type, and extent of the lesions. Subsequent studies ${ }^{11-15}$ did, however, describe c-PVL in more detail, taking the site and extent of the lesion into account and relating these findings to the neurodevelopmental outcome. The data of most of these studies were recently analysed together by Holling and Leviton, ${ }^{16}$ who reported a prevalence of cerebral palsy in only $35 \%$ of infants with "small lesions".

The aim of this study was to compare the US evolution and the neurodevelopmental outcome of infants with grade II and III PVL.

\section{Methods}

Between 1990 and 1998 all preterm infants ( $n=3451)$ of 32 weeks gestation or less, admitted to the level III neonatal intensive care units of Lille and Utrecht, were enrolled in a prospective cranial ultrasound (US) study.

\section{CRANIAL US ASSESSMENT}

Cranial US was performed as soon as possible after admission to the neonatal unit, using a Kontron Sigma 1AC until 1993 and subsequently a Vingmed Diasonics in Lille and an ATL UM-4 in Utrecht with a 5-7.5-10 MHz 

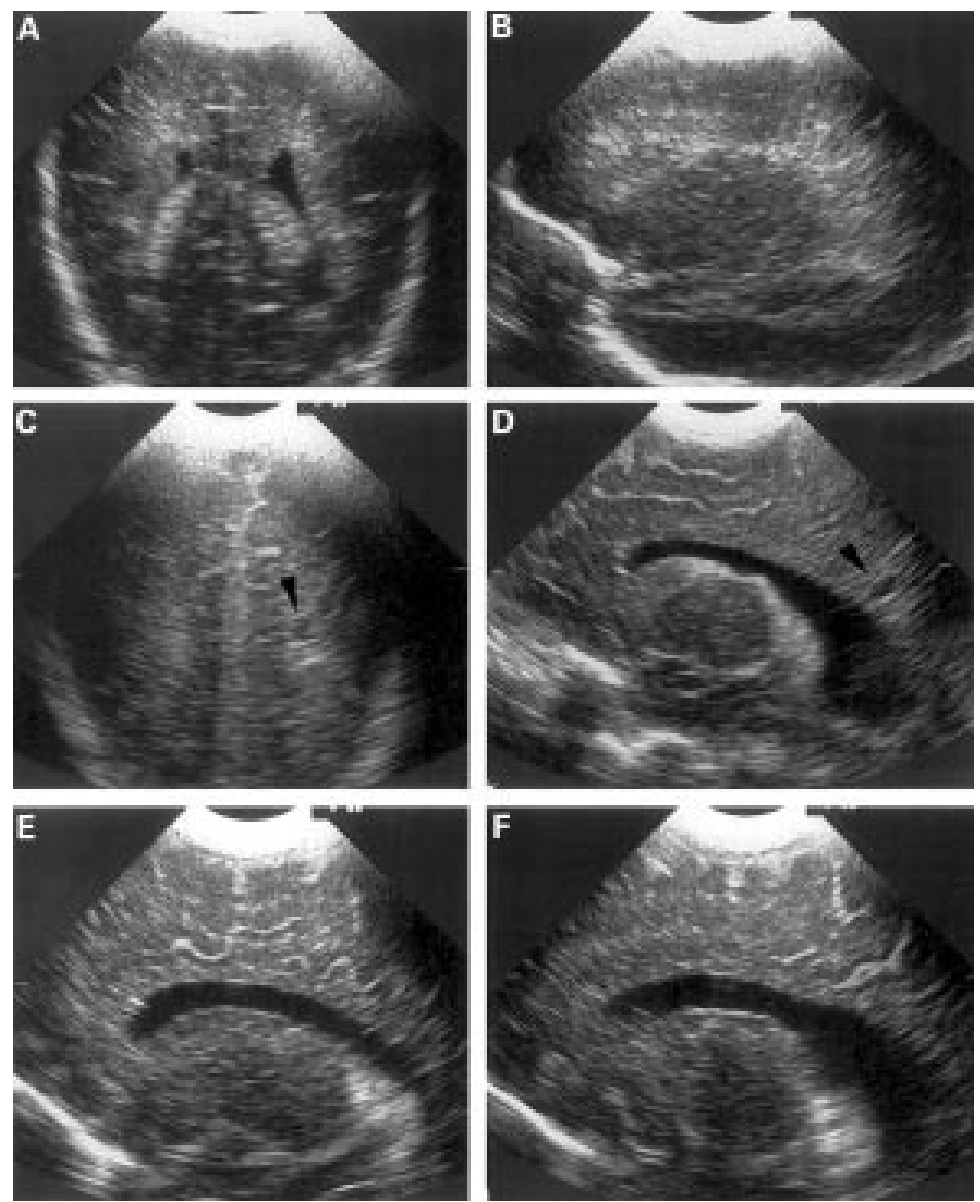

Figure 1 Coronal $(A)$ and left parasagittal $(B)$ views on day 4 in a preterm infant born at 29 weeks gestation, showing mild symmetrical periventricular densities. At 6 weeks of age, the coronal (C) and left parasagittal (D) views show a localised left sided occipital cyst (arrowhead) with associated mild ventriculomegaly. At 40 weeks postmenstrual age, in the right $(E)$ and left $(F)$ parasagittal views, the cyst is no longer seen and ventriculomegaly is only present on the left.
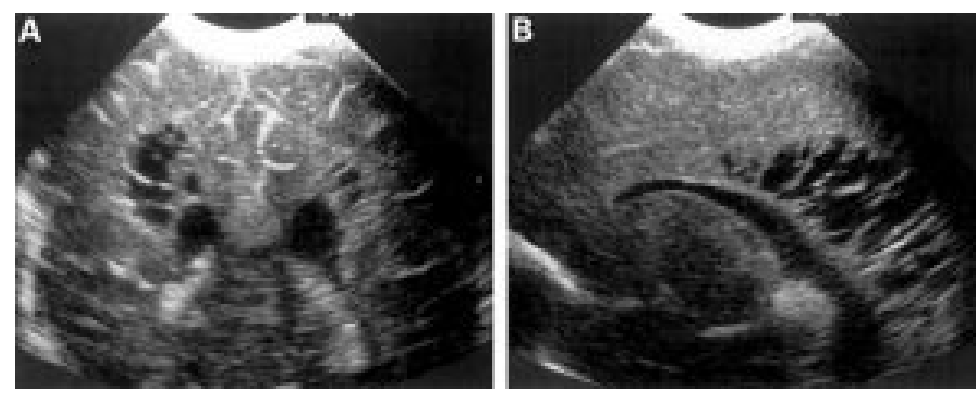

Figure 2 Coronal $(A)$ and right parasagittal (B) views on day 24 in a preterm infant born at 28 weeks gestation, showing extensive cysts, more on the right than on the left side.

Table 1 Clinical and outcome data for infants with grade II and grade III periventricular leucomalacia

\begin{tabular}{llll}
\hline & $\begin{array}{l}\text { Grade II } \\
(n=39)\end{array}$ & $\begin{array}{l}\text { Grade III } \\
(n=39)\end{array}$ & OR (95\% CI) \\
\hline $\begin{array}{l}\text { Gestational age (weeks) } \\
\text { Birth weight (g) }\end{array}$ & $\begin{array}{l}29(1.8) \\
1292(321)\end{array}$ & $\begin{array}{l}29(1.8) \\
1204(306)\end{array}$ & \\
$\begin{array}{l}\text { Diagnosis } \\
\text { During the 1st month }\end{array}$ & $18(47 \%)$ & $24(77 \%)$ & 3.81 (1.19 to 12.63) \\
$\quad \begin{array}{l}\text { After the 1st month } \\
\text { Unknown }\end{array}$ & 20 & 7 & \\
Outcome & 1 & 8 & \\
$\quad$ Death & 1 & 12 & \\
$\quad$ Normal & $9(24 \%)$ & $1(3 \%)$ & \\
$\quad$ Cerebral palsy & 29 & 26 & \\
$\quad$ Independent walking & 22 & 3 & \\
\hline
\end{tabular}

Values for gestational age and birth weight are mean (SD). transducer. Examinations were routinely performed two to three times during the first week of life and then once a week until discharge or more often as clinically indicated. Examinations were performed using the $7.5 \mathrm{MHz}$ transducer in both units. The $10 \mathrm{MHz}$ transducer was sometimes used when onset of cystic lesions was suspected. c-PVL was diagnosed as an area of increased echogenicity in the acute phase with a subsequent breakdown into echolucent areas within the following two to six weeks. c-PVL was classified according to the grading system of de Vries et $a l .{ }^{5}$ Grade II leucomalacia was present if there were transient periventricular densities, evolving into small localised cysts (fig 1). Grade III leucomalacia was defined as areas of periventricular densities evolving into extensive periventricular cystic lesions (fig 2). All US pictures were independently reported by neonatologists (VP, CD, LdV) from both neonatal units, and agreement on classification was reached in all but two cases, which were subsequently excluded.

NEUROLOGICAL ASSESSMENT OF OUTCOME Assessment of outcome was made on clinical examination at discharge using the standardised protocol of Amiel-Tison and Grenier ${ }^{17}$ in Lille and the Dubowitz assessment ${ }^{18}$ in Utrecht. Children were subsequently assessed at $6,9,12,18$, and 24 months corrected age, using items from Amiel-Tison and Stewart ${ }^{19}$ and Touwen. ${ }^{20}$ Cerebral palsy was classified according to the criteria of Hagberg et al. ${ }^{21}$

\section{STATISTICAL ANALYSIS}

The results of all comparisons are presented as relative risks (RR), odds ratios (OR), and 95\% confidence intervals in parentheses.

\section{Results}

During the study period, c-PVL was diagnosed in 96 infants $(2.8 \%)$. There was no significant difference in the prevalence between the centres (Lille 57/1824, 3.1\%; Utrecht 39/1627, $2.4 \%$; OR $=1.31(0.85$ to 2.03$))$. Grade II PVL was more often diagnosed in Lille (30/57) than in Utrecht (9/39) (OR $=3.89$ (1.33 to 11.66)). Eighteen of the 96 infants were excluded from the study for the following reasons: seven had cysts on the first scan and were therefore considered to be of antenatal onset; nine were admitted after the first month of life with evidence of cysts on the first scan; no definite classification could be achieved in two cases. Of the remaining 78 infants, 39 had a grade II PVL and 39 had a grade III PVL. The two populations of grade II and grade III PVL were comparable with respect to gestational age and birth weight (table 1). The prevalence of both grade II and grade III PVL did not change with time.

TIMING AND EVOLUTION OF THE CYSTIC LESIONS Figure 3 reports the age in days when the cysts in grade II and grade III PVL were first noted. Nine infants were not scanned on a regular enough basis to time the onset of the cysts. 


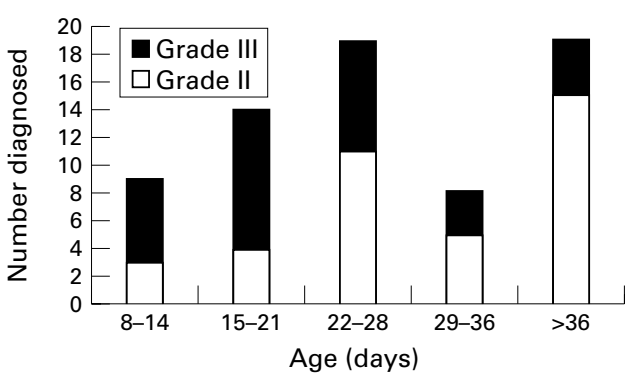

Figure 3 The age in days when the cysts were first diagnosed is shown for children with grade II and grade III periventricular leucomalacia.

Grade II $(n=39)$

Exact timing was not possible in only one infant. The echodensities were present at birth in all but one of the children. Cysts developed within the first 4 weeks of life in 18 of the 38 infants and in 20 infants at a later stage. The mean (SD) age when the cysts were diagnosed, following the development of echodensities, was 35 (16) days. Localisation of the cysts was in the parieto-occipital region in 8/39 infants. The frontal or parietal white matter was affected in 24 cases, and in seven infants the cysts were diagnosed in the occipital periventricular white matter. The cysts were unilateral in 21/39 infants, following the presence of bilateral echodensities. In the infants with unilateral cysts, the hyperechogenicity was usually asymmetrical, being more pronounced on the side where the cysts were subsequently seen. One infant was not seen at 40 weeks postmenstrual age (PMA) for a repeat US examination. In the other 38 infants, scanned at 40 weeks PMA, the cysts were no longer seen using US in 13, and mild ventriculomegaly was the only sequel present at this stage in nine. Ventriculomegaly was also present in four other infants in whom the cysts could still be seen.

Grade III $(n=39)$

Exact timing was not possible in eight of the 39 infants. In all but seven cases, the echodensities were present at birth. Cysts developed within the first 4 weeks of life in 24 of the 31 infants and in seven at a later stage. The mean (SD) age when the cysts were first seen, following the appearance of echodensities, was 21 (10) days. The cysts were bilateral in all infants. Cysts were in the parieto-occipital region in 32/39 infants. In 20/32, the cysts extended into the frontal periventricular white matter. In the other seven infants, cysts were located in the frontoparietal $(n=6)$ or parietal area $(n=1)$. Twelve infants died before 40 weeks PMA. In two of the remaining 27 infants, cysts were no longer seen at 40 weeks PMA, using US, but ventriculomegaly was now noted to be present. Mild ventriculomegaly was also diagnosed in 17 infants in whom cysts could still be diagnosed.

\section{NEURODEVELOPMENTAL OUTCOME}

Grade II

One infant died during the first year of life, after discharge home. Twenty nine infants developed cerebral palsy, eight quadriplegia,
15 diplegia, and six hemiplegia. Twenty two of these infants have so far achieved independent walking at 18-30 months. All but one of the neonates with quadriplegia had parietal cysts, associated with frontal or occipital cysts in seven. The last one had occipital cysts only. Nine infants had transient tone abnormalities, but were considered to be normal at 2 years. Cysts were frontal in one of them, parietal in four, and occipital in four. Fourteen infants with unilateral cysts developed cerebral palsy, which was bilateral in nine of them: five hemiplegia, six diplegia, and three quadriplegia. Among the nine children who had no cysts around term but who had developed mild ventriculomegaly, seven developed cerebral palsy, and two were considered to be normal. One of these two had frontal cysts and the other one had small occipital cysts. Three of the other four infants, with a normal US scan at discharge - that is, no cysts and no ventriculomegaly-were normal at follow up, and one developed a hemiplegia.

\section{Grade III}

All but one of the 27 survivors with grade III PVL developed cerebral palsy. They were all associated with severe handicap and only three of them, with spastic diplegia, have so far achieved independent walking at 3-5 years. The other 23 had quadriplegia with severe restriction of spontaneous mobility. The last infant with extensive frontal cysts and localised occipital cysts did not develop cerebral palsy, but has a pronounced squint and learning disabilities, with a developmental quotient $<70$ at 24 months corrected age.

\section{Comparison of grade II and grade III PVL}

Cysts were noted to develop more often after the first month of life (53\%) in infants with grade II PVL than in those with grade III PVL (22\%) (OR 3.81 (1.19 to 12.63)). Cysts were unilateral in 21 of the 39 infants with grade II PVL, whereas this was never seen in grade III PVL (OR indefinite; RR 3.17 (2.16 to 4.64)). At 40 weeks PMA, cysts were no longer seen using US in 13/38 infants with grade II PVL, with ventriculomegaly being the only visible sequel in nine cases. In grade III PVL, cysts were still present in 25 of the 27 surviving infants.

Nine infants with grade II PVL were free of motor sequelae at follow up compared with only one infant with grade III PVL (OR 8.07 (0.92 to 181.66$)$ ). Twenty two of 29 children with grade II PVL who developed cerebral palsy achieved independent walking compared with 3/26 with grade III PVL (OR 75 (11.4 to 662)).

\section{Discussion}

In this study, the US diagnosis and subsequent neurodevelopmental outcome of 39 infants with grade II PVL are reported and these data are compared with the data of a group of 39 infants with grade III PVL. In the cohorts described, the prevalence of c-PVL was low 
(2.8\%) and comparable in Lille and Utrecht. The prevalence usually varies from one centre to the next and is classically related to diagnosing periventricular haemorrhagic venous infarction as PVL. Bass et al, $^{22}$ being careful to differentiate between these two conditions, found a prevalence of $3.6 \%$ for PVL in a cohort of neonates with a birth weight below $1500 \mathrm{~g}$. Perlman et $a l^{23}$ reported a prevalence of $2.3 \% \mathrm{c}-\mathrm{PVL}$ in a cohort of neonates of less than $1750 \mathrm{~g}$, although late onset c-PVL could have been missed, as US was not obtained routinely after day 28 . Zupan et $a l^{24}$, using a US protocol comparable with the one used in this study, observed a higher incidence of $9.2 \%$ in a cohort of 802 infants born between 24 and 32 weeks. Baud et al ${ }^{25}$ have shown considerable incidence differences among centres and suggested that the hospital recruitments and policies should be reported in more detail.

No distinction was made between grade II and grade III PVL in any of these recent studies. We were not able to explain the difference in the prevalence of grade II and grade III between our two units. Although different US machines were used, the two units had the same protocol, preferably using a $7.5 \mathrm{MHz}$ transducer. We did not observe any variation with time in the prevalence of both grade II and grade III PVL, although appreciable changes in the care of low birthweight infants has been noted during the last 10 years. This supports the recent hypothesis that the pathophysiological cascade leading to PVL may be initiated before birth. ${ }^{26} 27$

Although according to our protocol the infants were only scanned once a week, we found a significant difference between the time when the cysts were first diagnosed. We could, however, not be more precise about the exact day when the cysts developed because of the limited number of US examinations. In infants with grade II PVL, cysts were noted to develop more often after the first month of life, in contrast with those with grade III PVL cases, who tended to develop cysts within the first 2-3 weeks of life. All seven infants with grade III PVL who developed cysts after day 28 had so called "late onset PVL". ${ }^{28}{ }^{29}$ The later development of cysts in grade II PVL was not due to late onset PVL, as echodensities were present on the first or second scan in all but one of the infants. Attention was previously paid to late onset PVL, often related to septicaemia or necrotising enterocolitis, and it was suggested that repeat US examinations should be performed after a second period of severe illness. ${ }^{28}{ }^{29}$ Attention has so far not been drawn to the later occurrence of cysts in infants with grade II PVL, defined as PVL with smaller and more localised cysts compared with grade III PVL. In addition to the cysts being smaller, they are only present for a short time and it is not uncommon that they are no longer detectable by ultrasonography at 40 weeks PMA. Mild ventriculomegaly will then be the only sequel visible at this stage. These smaller cysts can therefore be easily missed, when infants

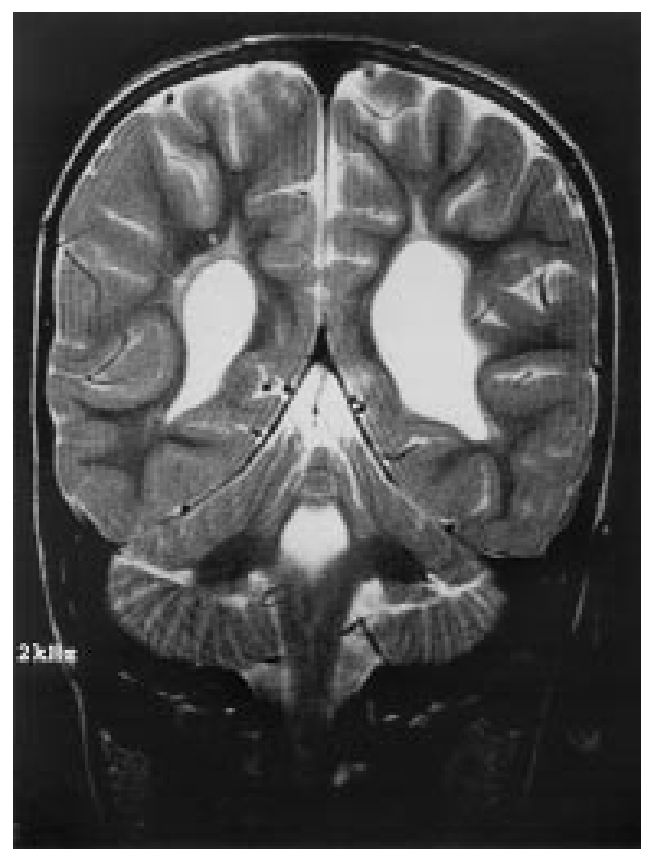

Figure 4 Magnetic resonance image of the infant in fig 1, performed at 2 years of age. The T2 weighted SE sequence shows asymmetrical ventriculomegaly, more pronounced and irregular on the left, and bilateral gliosis (courtesy of Dr Bouman, Deventer).

are not scanned with a $7.5 \mathrm{MHz}$ transducer and/or discharged within the first 2-3 weeks of life.

Grade II PVL was unilateral in more than half of the cases $(54 \%)$ in spite of bilateral echodensities on US to start with. It was of interest that magnetic resonance imaging (MRI), performed in a small number of these cases, either in the neonatal period or at follow up, ${ }^{30}$ showed bilateral areas of abnormal signal intensity, suggestive of bilateral gliosis (fig 4).

The infants with grade III PVL always showed bilateral cysts. Most of the cystic lesions in grade III PVL (82\%) were diagnosed in the parieto-occipital periventricular white matter compared with $20 \%$ of the infants with grade II PVL. In the initial report of Fawer and Calame, ${ }^{6}$ the focal or small lesions were all in the frontal periventricular white matter, while the large lesions were found in the frontoparietal or frontoparieto-occipital periventricular white matter. In other studies, small lesions were observed in the different periventricular areas. ${ }^{12}{ }^{13}$ In fact, it seems that cysts in grade II PVL are more often diagnosed anteriorly but can also occur in the parietal or occipital periventricular white matter.

All but one of our infants with grade III PVL developed cerebral palsy and only three of them achieved independent walking. In comparison, cerebral palsy was diagnosed in 29/39 infants with grade II PVL but was less severe in this subgroup, as 22 achieved independent walking. Children with a normal outcome were more often present among those with grade II PVL and three of these nine infants did in fact have a normal US scan at 40 weeks PMA. In contrast with the infants with grade III PVL, no clear association could be found 
between the localisation of the cysts and the neurodevelopmental outcome in infants with grade II PVL.

The follow up data are in agreement with a recent review of the literature. ${ }^{16}$ In the pooled population described by Holling and Leviton, ${ }^{16}$ quadriplegia was associated with white matter lesions in frontal, parietal, or occipital lobes of both hemispheres. In contrast, only $35 \%$ of infants with "small" lesions and $61 \%$ with "medium" lesions developed cerebral palsy. Levene ${ }^{30}$ was the first to suggest that US scan performed around 40 weeks PMA has the best predictive value with regard to subsequent neurodevelopmental outcome. Recent studies $^{431}$ have focused on ventriculomegaly and suggested that it strongly indicates white matter disease. Paneth ${ }^{32}$ recently suggested inclusion of ventricular enlargement in the spectrum of white matter disease. It is quite possible that some of these infants with ventriculomegaly had grade II PVL, but that this diagnosis was not made because US examinations were performed during a limited number of weeks. In our cohort of infants with c-PVL, ventriculomegaly on its own was indeed a good predictor of cerebral palsy, as $29 / 30$ infants with ventriculomegaly around term developed cerebral palsy. It is, however, important to bear in mind that ventriculomegaly can also be present at term in infants who had an intraventricular haemorrhage during the neonatal period. In these infants, ventriculomegaly may be due to mild posthaemorrhagic ventricular dilatation, which is unlikely to lead to an adverse neurological outcome. ${ }^{6}$ It is sometimes suggested that the enlarged ventricles, belonging to the spectrum of white matter disease, are more irregular in shape. The distinction between these two entities, which can also occur together, can in our experience only be confidently made when long term sequential US studies are available.

With the increasing use of MRI during the early neonatal period, it has become clear that cranial ultrasonography underdiagnoses the milder or diffuse lesions of white matter disease in particular. ${ }^{303435}$ Sie et $a l^{36}$ recently showed that conventional MRI was superior in identifying and quantifying cerebral white matter damage in very low birthweight infants. Some $71 \%$ of our population with unilateral cysts developed cerebral palsy with bilateral motor signs in $64 \%$, compared with $76 \%$ and $55 \%$ in the pooled population described by Holling and Leviton. ${ }^{16}$ We were not able, however, to quantify the magnitude of hyperechogenicity, which could be more important than cysts in grade II PVL. Inder et $a l^{\beta 7}$ recently showed earlier detection of the diffuse component of c-PVL with diffusion weighted MRI than with conventional brain imaging techniques. Diffusion weighted MRI, used at an early stage, could prove to be more reliable in predicting whether areas of increased echogenicity on US will evolve into cysts. At present, however, MRI cannot replace cranial ultrasonography as a routine tool in most neonatal units. We therefore strongly recommend that weekly US examinations are performed in all at risk infants until discharge, even when no obvious densities are apparent on previous US examinations.

1 Dolfin T, Skidmore MB, Fong KW, et al. Diagnosis and evolution of periventricular leukomalacia: a study with real-time ultrasound. Early Hum Dev 1984;9:105-9.

2 Trounce JQ, Fagan D, Levene MI. Intraventricular haemorrhage and periventricular leukomalacia: ultrasound and autopsy correlation. Arch Dis Child 1986;61:1203-7.

3 Leviton A, Floyd G. Ventriculomegaly, delayed myelination, white matter hypoplasia and periventricular leukomalacia: how are they related? Pediatr Neurol 1996;15:127-36.

4 Ment L, Vohr B, Allan W, et al. The etiology and outcome of cerebral ventriculomegaly at term in very low birth weight preterm infants. Pediatrics 1999;104:243-8.

5 de Vries LS, Eken P, Dubowitz LMS. The spectrum of leukomalacia using cranial ultrasound. Behav Brain Res 1992;49:1-6.

6 Fawer CL, Calame A. Significance of ultrasound appearances in the neurological development and cognitive abilities of preterm infants at 5 years. Eur 7 Pediatr 1991; 150:515-20

7 Calvert SA, Hoskins EM, Fong KW, et al. Periventricular leukomalacia: ultrasound diagnosis and outcome. Acta Paediatr Scand 1986;75:489-96.

8 Cooke RWI. Early and late cranial ultrasonographic appearances and outcome in very low birthweight infants. Arch Dis Child 1987;62:931-7.

9 Graziani LJ, Pasto M, Stanley C, et al. Neonatal sonographic correlates of cerebral palsy in preterm infants. Pediatrics 1986;78:88-95

10 Fazzi E, Lanzi G, Gerardo A, et al. Neurodevelopmental outcome in very-low-birth-weight infants with or without periventricular haemorrhage and/or leucomalacia. Acta Paediatr 1992;81:808-11.

11 Graham M, Levene MI, Trounce JQ, et al. Prediction of cerebral palsy in very low birthweight infants: prospective ultrasound study. Lancet 1987;ii:593-6.

12 Monset-Couchard $M$, de Bethmann O, Radvanyi-Bouvet $\mathrm{MF}$, et al. Neurodevelopmental outcome in cystic periven-
tricular leukomalacia (CPVL) (30 cases). Neuropediatrics tricular leukomalaci

13 Ringelberg J, van de Bor M. Outcome of transient periventricular echodensities in preterm infants. Neuropediatrics $1993 ; 24: 269-73$

14 Fujimoto S, Yamaguchi N, Togari H, et al. Cerebral palsy of cystic periventricular leukomalacia in low-birth-weight infants. Acta Paediatr 1994;83:397-401.

15 Rogers B, Msall M, Owens T, et al. Cystic periventricular leukomalacia and type of cerebral palsy in preterm infants. f Pediatr 1994;125:S1-8

16 Holling EE, Leviton A. Characteristics of cranial ultrasound white-matter echolucencies that predict disability: a review. Dev Med Child Neurol 1999;41:136-9.

17 Amiel-Tison C, Grenier A. Neurological assessment during the first year of life. Oxford: Oxford University Press, 1986.

18 Dubowitz LMS, Dubowitz V. The neurological assessment of the preterm and full-term infant.Clinics in developmental of the preterm and full-term infant.Clinics in developmental Lippincott, 1981

19 Amiel-Tison C, Stewart A. Follow-up studies during the first five years of life: a pervasive assessment of neurological function. Arch Dis Child 1989;64:496-502.

20 Touwen BCL. Examination of the child with minor neurological dysfunction, 2nd Ed. Clinics in Developmental Medicine No 79. London: SIMP/Heinemann; Philadelphia: Lippincott, 1981.

21 Hagberg B, Hagberg G, Olow I. The changing panorama of cerebral palsy in Sweden 1954-1970. II. Analysis of various syndromes. Acta Paediatr Scand 1975;64:193-200.

22 Bass WT, Jones MA, White LE, et al. Ultrasonographic differential diagnosis and neurodevelopmental outcome of cerebral white matter lesions in premature infants. 7 Perinatol 1999;19:330-6.

23 Perlman JM, Risser R, Broyles RS. Bilateral cystic periventricular leukomalacia in the premature infant: associated risk factors. Pediatrics 1996;97:822-7.

24 Zupan V, Gonzalez P, Lacaze-Masmonteil T, et al. Periventricular leukomalacia: risk factors revisited. Dev Med Child tricular leukomalacia: ris

25 Baud O, Truffert P, Debillon T, et al. Enquête sur la mortalité et les complications neurologiques graves des grands prématurés dans cinq centres de niveau III. Arch Pediatr 1999;6:350.

26 Leviton A. Preterm birth and cerebral palsy: is tumor necrosis factor the missing link? Dev Med Child Neurol 1993;35:549-58.

27 Grether JK, Nelson KB. Maternal infection and cerebral palsy in infants of normal birth weight. $\mathcal{F} A M A$ 1997;278:207-11.

28 De Vries LS, Regev R, Dubowitz LM. Late onset cystic leukomalacia. Arch Dis Child 1986;61:298-9.

29 Debillon T, Zupan V, Magny JF, et al. Leucomalacie periventriculaire d'apparition tardive chez un prématuré. Arch Pediatr1993;50:671-4.

30 Levene MI. Cerebral ultrasound and neurological impairment: telling the future. Arch Dis Child 1990;65:469-71.

31 Kuban K, Sanocka U, Leviton A, et al. White matter disorders of prematurity: association with intraventricular hemorrhage and ventriculomegaly. F Pediatr 1999;134:539-46. 
32 Paneth N. Classifiying brain damage in preterm infants. $\mathcal{F}$ Pediatr 1999;134:527-9.

33 Van de $1999,134.527-9.0$. ultrasound and magnetic resonance imaging in predicting neurodevelopmental outcome in preterm infants. Pediatrics 1992;90:196-9.

34 De Vries LS, Groenendaal F, van Haastert IC, et al. Correlation between the degree of periventricular leukomalacia diagnosed using cranial ultrasound and MRI later in infancy in children with cerebral palsy. Neuropediatrics 1993;24:263-8.
35 Battin M, Maalouf EF, Counsell S, et al. Magnetic resonance imaging of the brain in very preterm infants: resonance imaging of the brain in very preterm infants:
visualisation of germinal matrix, early myelination, and visualisation of germinal matrix, early mye
cortical folding. Pediatrics 1998;101:957-62.

36 Sie LT, van der Knaap MS, van Wezel-Meijler G, et al. Early MR features of hypoxic-ischemic brain injury in neonates with periventricular densities on sonograms. AfNR Am f Neuroradiol 2000;21:852-61.

37 Inder T, Huppi P, Zientara G, et al. Early detection of periventricular leukomalacia by diffusion-weighted magnetic resonance imaging techniques. F Pediatr 1999;134:631-4.

\title{
Narrative Based Medicine, An Interdisciplinary Conference
}

\author{
Research, Narrative, and Practice
}

A two day conference-Monday 3rd and Tuesday 4th September 2001

Homerton College, Cambridge, UK

BMF Publishing Group

For full details contact: BMA/BMJ Conference Unit, Tavistock Square, London, WC1H 9JP Tel: +44 (0)20 7383 6819; fax: +44 (0)20 7383 6663; email: clyders@bma.org.uk. www.quality.bmipg.com 